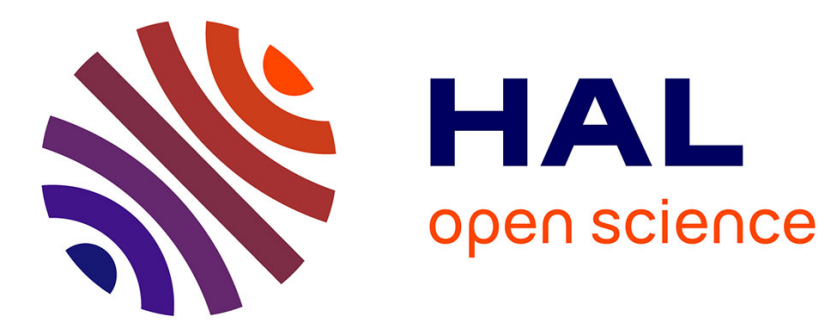

\title{
Clinical research in newborn infants: difficulties and specificity
}

Isabelle Ligi, Farid Boubred, Isabelle Grandvuillemin, Umberto Simeoni

\section{To cite this version:}

Isabelle Ligi, Farid Boubred, Isabelle Grandvuillemin, Umberto Simeoni. Clinical research in newborn infants: difficulties and specificity. European Journal of Clinical Pharmacology, 2010, 67 (S1), pp.2932. 10.1007/s00228-010-0921-3 . hal-00642437

\section{HAL Id: hal-00642437 https://hal.science/hal-00642437}

Submitted on 18 Nov 2011

HAL is a multi-disciplinary open access archive for the deposit and dissemination of scientific research documents, whether they are published or not. The documents may come from teaching and research institutions in France or abroad, or from public or private research centers.
L'archive ouverte pluridisciplinaire HAL, est destinée au dépôt et à la diffusion de documents scientifiques de niveau recherche, publiés ou non, émanant des établissements d'enseignement et de recherche français ou étrangers, des laboratoires publics ou privés. 


\title{
Clinical research in newborn infants: Difficulties and specificity (TEDDY)
}

Isabelle Ligi, Farid Boubred, Isabelle Grandvuillemin, Umberto Simeoni

Division of Neonatology, La Conception Hospital, Assistance Publique-Hôpitaux de Marseille and Faculté de Médecine, Université de la Méditerranée, Marseille, France

Correspondence: Prof Umberto Simeoni, Division of Neonatology, La Conception

Hospital, AP-HM, 147 Boulevard Baille, 13385 Marseille, France.

Tel : + 33491384041

Fax : + 3349138404

Umberto.simeoni@ap-hm.fr

\begin{abstract}
Clinical trials are essential in neonates to evaluate scientifically the efficacy and safety of drugs. Neonatal specificities that induce specific obstacles in neonatal studies are detailed. This review looks also at new recommendations recently developed by the European Commission to promote a safe and ethical research in neonatology.

\section{Keywords}

Clinical trial, ethics, European legislation, neonatology

\section{INTRODUCTION}

Clinical trials are essential to provide a safe, effective and evidence based medicine. In contrast, up to $90 \%$ of drugs are used in an unauthorised and off-label manner in neonates with a greater risk of drug toxicity [1]. Actually, reserves to perform clinical trials in neonates are bound to physiological and ethical specifics. Indeed, neonatal studies encompass numerous obstacles of which the vulnerability and the incompetence
\end{abstract}


of this population, the technical difficulties, the uniqueness of neonatal diseases and the frequency of emergencies situations. Neonatology is a relatively new discipline that should largely benefit from clinical research but the fragility of neonatal subjects makes them highly vulnerable to experimental interventions. That's why specific risks and limits of studies in neonatal period must be counter-balanced by both a strictly controlled but also incited research. Recently, the European Commission has edited recommendations on clinical trials in pediatrics in a way to regulate, promote and harmonize research in the european pediatric population [2]. This paper focuses on methodological and ethical problems specific to clinical trials in neonates.

\section{SAFETY AND RISK}

Children are not small adults and neonates are not small children. History of clinical research is full of dramatic examples that demonstrate the uniqueness of neonatal physiology. Risk/benefits equipoise must always be considered [3, 4].

\section{Physiologic specificity of the neonates}

Neonates are the group of children from birth up to 28 days excluded, be they preterm or term. This transitional period is characterized with physiological immaturity of many organs or systems that could impact the pharmacokinetics, pharmacodynamics and finally the tolerance of drugs [5]. Cardiopulmonary changes are a critical event that could influence organ functions (renal blood flow, brain perfusion, hepatic metabolism). In preterm babies, many pathological situations (as patent ductus arteriosus or respiratory distress syndrome) can interfere with a trial protocol and have to be considered. Another aspect of neonatal specificity is brain development. Indeed, maturation of central nervous 
system is a continuous process (glial multiplication) that completes long after birth (one year of age). This immaturity makes the brain more vulnerable so as autoregulation of cerebral blood flow is limited. Drugs can interact with neurotransmitters and lead to long term neurodevelopmental effects. In particular, drugs interacting with bilirubin metabolism should be carefully monitored. Renal function is also altered in neonates. Glomerular filtration is physiologically reduced. This impairment is accentuated by preterm birth (incomplete nephrogenesis before 34 weeks of post-menstrual age) or by ongoing disease or intrauterine growth retardation. So, the lower renal elimination capacity of neonates has to be considered and estimated both with post-natal and postmenstrual ages. The pharmacokinetics of drugs can be also changed by hepatic and enzymatic immaturity that altered hepatic clearance as well as glucuronidation and enterohepatic recirculation. Moreover, the immaturity of gastrointestinal tract (high gastric $\mathrm{pH}$, reduced pancreatic and biliary functions) can alter the bioavailability of orally administrated drugs and make it unpredictable. Gastrointestinal immaturity makes the preterm neonate more susceptible to necrotizing enterocolitis. Lastly, associated diseases (respiratory distress syndrome, patent ductus arteriosus, necrotizing enterocolitis or retinopathy of prematurity) and conditions (prematurity or in utero growth restriction) can independently affect the pharmacokinetics and pharmacodynamics of drugs.

\section{Risks of clinical trials in neonates}

The immaturity of neonates associated with severe diseases that require multiple drugs administration increases the risk of iatrogenic events, drug interactions and adverse reactions. In a recent review, Sammons and colleagues evaluated the drug toxicity studying 739 pediatric clinical trials published over 7 years [6]. Seventy one percent of 
trials reported adverse events and twenty percent reported serious adverse events. In eleven percent of the trials, moderate or severe adverse drug reactions (ADR) were present. Thirteen percent of trials involved neonates, but neonatal trials were overrepresented in studies with severe ADR (9 in 35 trials). The severe ADR included apnea, hypotension, gastrointestinal hemorrhage, ototoxicity and sepsis. Mortality was also higher in trials involving neonates even if mortality was often linked to the natural process of the disease. In two trials in neonates, mortality was higher in the treatment group. But surprisingly, the authors noticed that only $2 \%$ of the trials had an independent safety monitoring committee as recommended in Europe. Another peculiarity of neonatal research is the necessity of long term follows up for safety. Indeed, many studies have shown that early infancy is a critical window for the programming of physiological changes. We have to consider the possibility of long term effects, in particular neurodevelopmental effects of drugs used in neonatal period.

\section{Pain and stress}

Many studies showed that repeated or prolonged exposure to pain or stress is an independent risk factor for brain damage and neurodevelopment impairment [7, 8]. Indeed, immature neurons and glial elements are vulnerable to apoptosis or excitotoxicity that may be favored by painful procedures. Limitation of stressing procedures (noise, light, blood sampling and investigations) must be a priority during clinical trials. Many validated non-invasive techniques (cardiac monitoring, transcutaneous measurement of pCO2, oxygen saturation from pulse oxymetry, cardiac and brain ultrasonography) can be used to evaluate clinical parameters. Pain has to be prevented, monitored using validated scales and adequately managed using pre-emptive treatment like sucrose or topical 
anesthesia. The use of indwelling catheter (umbilical or arterial venous catheter) has to be considered when repeated blood samples are needed.

\section{TRIALS DESIGN: METHODOLOGY, LIMITS}

The specificity of neonatal period has been emphasized in the previous sections and summarized in table 1 . The specificity of neonatal patients induces limitations in trials design that can be encompassed by methodological adaptations.

\section{Specific institutions for specific patients}

Special patients need special trials by experienced centers. These imply a specific regulation and representation of pediatric and neonatal medicine in institutional review boards and ethics committees as well as a local specific institutional and administrative assistance with appropriate resources dedicated to neonatal clinical research. These conditions are essential to ensure optimal protection, respect and medical support adapted to neonates.

\section{Specific methods for specific patients}

Even if a relative homogeneity of patients and standardization of neonatal care are marked advantages in neonatal research, studies are limited by small samples of eligible patients and by a low number of perinatal centers. These obstacles can be by-passed by the optimization of design methods. Randomised, multicenter and blinded studies are the gold standard both for demonstration of efficacy and safety. In this vulnerable population, size sample should be as small as possible to demonstrate efficacy with sufficient statistical power. Adaptive bayesian sequential modeling or other models also developed in adults can be used to decrease the number of participants. Otherwise, the use of 
placebo has to be discussed. The use of placebo is restricted compared to adults or children studies but can be considered in the case of poor or questionable efficacy or safety of the commonly used treatment. In particular in studies on analgesia, the use of placebo is not acceptable. A comparator group is ethically more acceptable and clinically more relevant even in case of off-label use if they are the standard of care. The technique of population pharmacokinetics based on lower blood samples in a larger population is interesting to limit both blood samples and bias linked to the maturating process and inter-individual variability. Stratification by term gestation or birth weight of the trial population is often required to limit the influence of maturation process. Many antenatal (antenatal treatment, maternal diseases) and postnatal (drugs, patent ductus arteriosus, hemodynamic and respiratory status) conditions can affect outcome and require identification and careful analysis to limit bias. Relevant primary endpoints have to be described, based on harmonized definitions and assessed using validated procedures for judgment. The complications of prematurity (bronchopulmonary dysplasia, intraventricular haemorrhage, necrotizing enterocolitis, retinopathy of prematurity) and survival are classical secondary endpoints that need to be assessed. Lastly, we underline the importance of long-term physical and neurodevelopmental follow up to evaluate the long term impact of drugs in this maturating population.

\section{Specific drugs for specific patients}

Iatrogenic events are more frequent in neonates. These underline the importance of accurate choice of formulations and route of administration. First, they suffer more medication errors. The implication of drugs companies is essential to provide appropriate formulations in way to limit dilutions. Second, adverse drug reaction are more frequent 
and more severe in neonates. Potential incompatibilities, excessive amounts of electrolytes, cutaneous or digestive tolerance must be considered. Finally, it is essential to re-adjust dosage of drugs over time according to actual weight and organ maturation of the neonate to maintain the best efficacy and safety.

\section{Volume of blood samples}

Preterm and term neonates have restricted blood volume (80 to $90 \mathrm{ml} / \mathrm{Kg}$ ). Moreover, pathologic conditions increase blood loss and blood sampling necessity. Recommendations have been applied to limit trial-related blood loss. Micro-methods samples are required and limited to $2.4 \mathrm{ml}$ blood per $\mathrm{Kg}$ body weight for the $3 \%$ limit, over a 4-week period. Expected blood loss has to be detailed both in trial protocol and in parent information sheet.

\section{ETHICAL ISSUES}

Ethical principles in neonatal research are expressed in the Declaration of Helsinki and based on three foundations: respect for persons, beneficence and justice [9].

\section{Balance of risk and benefit}

Probability of benefits and potential risks need to be continuously balanced [10]. The neonate's interest always prevails over that of science or society. Risk is classically divided into three categories: minimal, low or minor and high. Risk also depends on the invasiveness of the procedures and monitoring or the toxicity of the drugs. Benefit can be defined as a progress in safety or efficacy in treatment. The risk/benefit balance must take into considerations the severity of the condition/disease studied, the vulnerability of the neonates and the expected improvement in comparison with an alternative existing 
treatment. Non-therapeutic experiments with benefits for the neonatal population have a limited place in neonatal research and can only be considered if they cannot be performed in older children with minimal or low risk. As in adults, risk has to be continuously monitored using a Data and Safety Monitoring Board. Independent paediatric experts are recommended.

\section{Ethical committee's composition}

Ethical committees are needed to provide an independent opinion on trial and on balance risk/benefits. The vulnerability and pharmacological peculiarities of neonates, the complexity and frequent emergency context of neonatal situations underline the importance to have a scrutiny scientific and ethical expertise of protocols. The committees must include paediatric physicians qualified in neonatal medicine and trained to clinical research as well as paediatric ethicists, pharmacologists or psychologists. In particular, they have to make sure that pain and stress induced by clinical research have been prevented and minimized [11]. They also must require minimal blood sampling [12].

\section{Parental consent}

As neonates can never obviously consent, the informed, voluntary and written consent of each legal representative of the neonate is ethically and legally required prior to enrollment. This consent meets the standards required in other consent. Information must be honest, adequate and must describe the aim and nature of the study, the expected benefits or potential risks, the name of investigators and contact details. The consent has to be given free from coercion. Sufficient time of thinking must be accorded and the possibility to revoke informed consent must be specified. Consent is a continuous process 
that needs time and multiple discussions during the trial. In practice, many circumstances could interfere with those consent criteria. Many concepts like randomization can be misunderstood by parents. Emergencies situations are frequent and can disturb consent process, parent objectivity particularly when time available to consent is short and diseases are life-threatening. Relationship between parents and the physician of their children is also complex. This relation depends of many psychological, emotional, cultural and social circumstances as well as severity of the disease of their children that could affect their judgment and influence their reasons to consent. Otherwise, it can be occasionally hard to respect parent decision that seems to compromise the fundamental rights of the infant to beneficence. Clinical research in neonates points out many philosophical questions and limits of legislation [13-15]. The important is to obtain the best consent possible and to adjust information honestly in a continuous and dynamic process of assent [16].

\section{CONCLUSION}

Authorities have now realized the importance of development of clinical trials peculiar to children. Recommendations have been made to harmonized practices over Europe in a way to promote a safe and ethical research. Guidelines reflect positive evolutions and changes of our societies on clinical research in paediatric population. However, applying these paediatric guidelines to neonatal research is sometimes challenging. These difficulties underline the necessity of specific protective regulations on neonatal research. Let us hope that pharmaceutical industry supports these changes. 


\section{REFERENCES}

1. Conroy, S., J. McIntyre, and I. Choonara, Unlicensed and off-label drug use in neonates. Arch Dis Child Neonatal Ed, 1999. 80: p. F142-145.

2. Sammons, H., Ethical considerations for clinical trials on medicinal products with the paediatric population. Recommendations of the Ad hoc group for the development of implementing guidelines for Directive 2001/20/EC relating to good clinical practice in the conduct of clinical trials on medicinal products for human use. http://ec.europa.eu/enterprise/pharmaceuticals/eudralex/vol10/ethical_considerations.pdf.

3. Sutherland, J., Fatal cardiovascular collapse of infants receiving large amounts of chloramphenicol. Am J Dis Child, 1959. 18: p. 761-767.

4. Silverman, W., et al., A different mortality rate and incidence of kernicterus among premature infants allotted to two prophylactic antibacterial regimens. Pediatrics, 1956. 18: p. 614-621.

5. Committee for medicinal products for human use. Guideline on the investigation of medicinal products in the term and preterm neonate. http://www.emea.europa.eu, 2007.

6. Sammons, H., et al., Safety in paediatric clinical trials - a 7-year review. Acta Paediatr, 2008. 97: p. 474-477.

7. Bartocci, M., et al., Pain activates cortical areas in the preterm newborn brain. Pain, 2006. 122: p. 109-117.

8. Anand, K. and R. Hall, Controversies in neonatal pain: an introduction. Semin Perinatol, 2007. 31: p. 273-274.

9. World Medical Assembly. The declaration of Helsinki. N Engl J Med, 1964. 271: p. 473-474.

10. Sammons, H., Ethical issues of clinical trials in children: a European perspective. Arch Dis Child, 2009. 94: p. 474-477.

11. Bellieni, C. and G. Buonocore, Recommendations for an ethical treatment of newborns involved in clinical trials. Acta Paediatr, 2010. 99: p. 30-32.

12. Choonara, I., Ethical and safety aspects of clinical trials in neonates. Earl Hum Dev, 20091. 85: p. S19-S20.

13. Modi, N., Ethical and legal issues in neonatal research. Semin Neonatol, 1998. 3: p. 303-314.

14. Stenson, B., C. Becher, and N. McIntosh, Neonatal research: the parental perspective. Arch Dis Child Fetal Neonatal Ed, 2004. 89: p. F321.

15. Allmark, P. and M. Spedding, Clinical trials in neonates: Ethical issues. Semin Fetal Neonatal Med, 2007. 12: p. 318-323.

16. Golec, L., et al., Informed consent in the NICU setting: an ethically optimal model for research solicitation. J Perinatol, 2004. 12: p. 783-791. 
Table 1. Neonatal specificities that impact on trial design.

- Organ/system immaturity

- Frequency of emergency context

- Limited number of eligible subjects and perinatal centers

- Increased iatrogenic risk

- Limited biologic sampling facilities and vascular access

- Influence of developmental specifics on outcome measurements 